\title{
About the Non-relativistic Structure of the Conformal Algebra
}

\author{
G. Burdet, M. Perrin, and P. Sorba \\ Centre de Physique Théorique, C.N.R.S., \\ Marseille Cedex 2, France
}

Received March 5, 1973

\begin{abstract}
A new basis of the conformal algebra is proposed, which makes appear two conjugated Schrödinger algebras. This basis allows to exhibit a chain which does not contain the Poincare algebra, between the $\mathscr{S} O(4,2)$ algebra and the (two-dimensional) extended Galilean one. This non-relativistic structure of the conformal algebra is well adapted to discuss some extreme models of hadrons based on collinear massless particles.
\end{abstract}

The existence of an isomorphism between a subgroup of the Poincaré group and the two-dimensional Galilean group is a well-known fact which clearly appeared in the "infinite-momentum" or "light-cone" formalism of high energy physics and led a "potential theoretic intuition" in relativistic quantum field theory and quantum electrodynamics.

More recently this Galilean structure in the "transverse plane" was used to derive the phenomenological Parton model of Björken and Feynman [1].

In this paper we show that the non-relativistic structure of the transverse plane again emerges in the conformal algebra which contains a subalgebra isomorphic to the two-dimensional Schrödinger algebra. We deduce that to any relativistic massless particle can be associated a non-relativistic one having a variable mass but without the potential energy present in the massive relativistic case.

Moreover we show that the two-dimensional Schrödinger algebra is the stability algebra of the "color" of the lightlike states introduced by Domokos [2] and we discuss the extreme collinear free massless quarks model of hadrons considered by Del Giudice et al. [3].

It is well-known that the d'Alembert equation is invariant under the conformal group which contains as a subgroup the Poincare transformations, invariance group of the Klein-Gordon equation. The conformal Lie algebra is isomorphic to the $\mathscr{S O}(4,2)$ algebra generated by the 15 skew-symmetric $\boldsymbol{M}_{\mu v}\left(\mu, v=0,0^{\prime}, 1,2,3,4\right)$ satisfying the following commutation relations:

$$
\left[M_{\mu v}, M_{\varrho \sigma}\right]=i\left(g_{\mu \sigma} M_{v \varrho}+g_{v \varrho} M_{\mu \sigma}-g_{v \sigma} M_{\mu \varrho}-g_{\mu \varrho} M_{v \sigma}\right)
$$


with

$$
\boldsymbol{g}_{00}=\boldsymbol{g}_{0^{\prime} 0^{\prime}}=-\boldsymbol{g}_{11}=-\boldsymbol{g}_{22}=-\boldsymbol{g}_{33}=-\boldsymbol{g}_{44}=1 \text {. }
$$

The generally used basis of the conformal algebra makes appear:

- A Lorentz algebra generated for example by the $\boldsymbol{M}_{\mu \nu}$ such that $\mu, v=0,1,2,3$.

- The corresponding translational and special conformal Abelian algebras

$$
\boldsymbol{p}_{\mu}=\boldsymbol{M}_{0^{\prime} \mu}+\boldsymbol{M}_{4 \mu} \quad \text { and } \quad \boldsymbol{c}_{\mu}=\boldsymbol{M}_{0^{\prime} \mu}-\boldsymbol{M}_{4 \mu} \quad \text { where } \quad \mu=0,1,2,3
$$

which respectively form with $\mathscr{S} \mathcal{O}(3,1)$ two conjugated Poincaré algebras.

- The dilatation $\boldsymbol{M}_{0^{\prime} 4}$ which commutes with the Lorentz part.

In fact another decomposition of the $\mathscr{S} \mathscr{O}(4,2)$ algebra can be given which exhibits its non-relativistic structure.

First one finds a subalgebra isomorphic to the two-dimensional extended Galilean algebra $\tilde{\mathscr{G}}_{2}$, the situation of which in the Poincaré algebra has been widely used. The generators are:

$$
\begin{aligned}
& \boldsymbol{H}=\frac{1}{2}\left(\boldsymbol{p}_{0}-\boldsymbol{p}_{3}\right)=\frac{1}{2}\left(\boldsymbol{M}_{0^{\prime} 0}+\boldsymbol{M}_{40}-\boldsymbol{M}_{0^{\prime} 3}-\boldsymbol{M}_{43}\right), \quad \boldsymbol{J}_{3}=\boldsymbol{M}_{12} \\
& \boldsymbol{P}_{\boldsymbol{j}}=\boldsymbol{p}_{\boldsymbol{j}}=\boldsymbol{M}_{0^{\prime} j}+M_{4 j}, \quad K_{j}=-\left(M_{0 j}+M_{3 j}\right), \quad j=1,2 \\
& \boldsymbol{M}=\boldsymbol{p}_{0}+\boldsymbol{p}_{3}=M_{0^{\prime} 0}+\boldsymbol{M}_{40}+\boldsymbol{M}_{0^{\prime} 3}+\boldsymbol{M}_{43}
\end{aligned}
$$

with the following non-zero commutation relations

$$
\begin{array}{ll}
{\left[\boldsymbol{J}_{3}, \boldsymbol{P}_{\boldsymbol{j}}\right]=\boldsymbol{i} \varepsilon_{3 \boldsymbol{j} \boldsymbol{k}} \boldsymbol{P}_{\boldsymbol{k}},} & {\left[\boldsymbol{J}_{3}, \boldsymbol{K}_{\boldsymbol{j}}\right]=\boldsymbol{i} \varepsilon_{3_{\boldsymbol{j} \boldsymbol{k}}} \boldsymbol{K}_{\boldsymbol{k}}, \quad \boldsymbol{j}, \boldsymbol{k}=1,2} \\
{\left[\boldsymbol{H}, \boldsymbol{K}_{\boldsymbol{j}}\right]=-\boldsymbol{i} \boldsymbol{P}_{\boldsymbol{j}},} & {\left[\boldsymbol{K}_{\boldsymbol{j}}, \boldsymbol{P}_{\boldsymbol{k}}\right]=\boldsymbol{i} \delta_{\boldsymbol{j} \boldsymbol{k}} \boldsymbol{M} .}
\end{array}
$$

Recall in particular that $\left\{\boldsymbol{K}_{\boldsymbol{j}}, \boldsymbol{P}_{\boldsymbol{j}}, \boldsymbol{M}\right\}$ form a two-dimensional Heisenberg algebra $\mathscr{H}_{2}$, and that this choice of the generators of $\tilde{\mathscr{G}}_{2}$ corresponds to the usual change of basis used in the infinite momentum formalism.

Secondly, $\tilde{\mathscr{G}}_{2}$ is embedded in the larger two-dimensional extended Schrödinger algebra $\tilde{\mathscr{S}}_{2}$ which corresponds to the largest group of the Newtonian space-time transformations leaving invariant the twodimensional Schrödinger equation [4]. This algebra is 9-dimensional and a basis can be given by adding to (2) the two generators

$$
\begin{aligned}
& \boldsymbol{C}=\frac{1}{2}\left(\boldsymbol{c}_{0}+\boldsymbol{c}_{3}\right)=\frac{1}{2}\left(\boldsymbol{M}_{0^{\prime} 0}-\boldsymbol{M}_{40}+\boldsymbol{M}_{0^{\prime} 3}-\boldsymbol{M}_{43}\right) \\
& \boldsymbol{D}=\boldsymbol{M}_{0^{\prime} 4}-\boldsymbol{M}_{03} .
\end{aligned}
$$

We have to add to (3) the following commutation relations

$$
\begin{array}{lll}
{\left[D, P_{j}\right]=-i P_{j},} & {\left[D, K_{j}\right]=i K_{j},} & {[D, H]=-2 i \boldsymbol{H},} \\
{\left[C, P_{j}\right]=i K_{j},} & {[C, H]=-i D,} & {[D, C]=2 i C .}
\end{array}
$$

It must be noticed that $\boldsymbol{H}, \boldsymbol{C}, \boldsymbol{D}$ generate an algebra isomorphic to $\mathscr{S} \mathscr{U}(1,1)$. 
Finally we can complete a basis of the $\mathscr{S} \mathcal{O}(4,2)$ algebra with another Heisenberg algebra $\mathscr{H}_{2}^{*}$ generated by

$$
\begin{gathered}
P_{j}^{*}=M_{0 \dot{j}}-M_{3 j}, \quad K_{j}^{*}=c_{j}=M_{0^{\prime} j}-M_{4 j}, \\
M^{*}=c_{0}-c_{3}=M_{0^{\prime} 0}-M_{40}-M_{0^{\prime} 3}+M_{43}
\end{gathered}
$$

and a $15^{\text {th }}$ generator $\Lambda=-\left(\boldsymbol{M}_{0^{\prime} 4}+\boldsymbol{M}_{03}\right)$ which plays the role of a dilatation or scale transformation

$$
\begin{array}{ll}
{\left[\Lambda, \boldsymbol{P}_{\boldsymbol{j}}\right]=\boldsymbol{i} \boldsymbol{P}_{\boldsymbol{j}},} & {\left[\Lambda, \boldsymbol{P}_{\boldsymbol{j}}^{*}\right]=-\boldsymbol{i} \boldsymbol{P}_{\boldsymbol{j}}^{*},} \\
{\left[\Lambda, \boldsymbol{K}_{\boldsymbol{j}}\right]=i \boldsymbol{K}_{\boldsymbol{j}},} & {\left[\Lambda, \boldsymbol{K}_{\boldsymbol{j}}^{*}\right]=-\boldsymbol{i} \boldsymbol{K}_{\boldsymbol{j}}^{*}, \quad \boldsymbol{j}=1,2,} \\
{\left[\Lambda, \boldsymbol{M}^{\prime}=2 \boldsymbol{i} \boldsymbol{M},\right.} & {\left[\Lambda, \boldsymbol{M}^{*}\right]=-2 \boldsymbol{i} \boldsymbol{M}^{*} .}
\end{array}
$$

Between the two Heisenberg algebras one finds the commutation relations

$$
\begin{array}{ll}
{\left[\boldsymbol{P}_{\boldsymbol{j}}, \boldsymbol{P}_{\boldsymbol{k}}^{*}\right]=2 i \delta_{\boldsymbol{j} \boldsymbol{k}} \boldsymbol{H},} & {\left[\boldsymbol{K}_{\boldsymbol{j}}, \boldsymbol{K}_{\boldsymbol{k}}^{*}\right]=2 i \delta_{\boldsymbol{j} \boldsymbol{k}} \boldsymbol{C},} \\
{\left[\boldsymbol{P}_{\boldsymbol{j}}, \boldsymbol{K}_{\boldsymbol{k}}^{*}\right]=-2 \boldsymbol{i} \varepsilon_{\boldsymbol{j} \mathbf{k} 3} \boldsymbol{J}_{3}-i \delta_{j k}(D-\Lambda),} & {\left[\boldsymbol{P}_{\boldsymbol{j}}^{*}, \boldsymbol{K}_{\boldsymbol{k}}\right]=2 \boldsymbol{i} \varepsilon_{\boldsymbol{j} \boldsymbol{k} 3} J_{3}+\boldsymbol{i} \delta_{j \boldsymbol{k}}(\boldsymbol{D}+\Lambda),} \\
{\left[\boldsymbol{P}_{\boldsymbol{j}}, \boldsymbol{M}^{*}\right]=2 \boldsymbol{i} \boldsymbol{P}_{\boldsymbol{j}}^{*},} & {\left[\boldsymbol{K}_{\boldsymbol{j}}, \boldsymbol{M}^{*}\right]=2 \boldsymbol{i} \boldsymbol{K}_{\boldsymbol{j}}^{*}} \\
{\left[\boldsymbol{P}_{\boldsymbol{j}}^{*}, \boldsymbol{M}\right]=-2 \boldsymbol{i} \boldsymbol{P}_{\boldsymbol{j}},} & {\left[\boldsymbol{K}_{\boldsymbol{j}}^{*}, \boldsymbol{M}^{*}=-2 \boldsymbol{i} \boldsymbol{K}_{\boldsymbol{j}}\right.}
\end{array}
$$

$$
\left[M, M^{*}\right]=-4 i \Lambda .
$$

Let us remark that $\boldsymbol{M}, \boldsymbol{M}^{*}, \Lambda$ also generate a 3-dimensional non-compact simple algebra. It is not necessary to write the commutation relations between the algebra $\mathscr{S} \mathcal{O}(2) \oplus \mathscr{S} \mathscr{U}(1,1)\left(\mathscr{S} \mathcal{O}(2)\right.$ generated by $\left.\boldsymbol{J}_{3}\right)$ and $\mathscr{H}_{2}^{*}$ because $\mathscr{S} \mathcal{O}(2) \oplus \mathscr{S} \mathscr{U}(1,1)$ acts on $\mathscr{H}_{2}^{*}$ exactly as on $\mathscr{H}_{2}$ and in fact one can form the two Schrödinger algebras

$$
\begin{aligned}
\tilde{\mathscr{S}}_{2} & =\mathscr{H}_{2} \square(\mathscr{S O}(2) \oplus \mathscr{S} \mathscr{U}(1,1)), \\
\tilde{\mathscr{S}}_{2}^{*} & =\mathscr{H}_{2}^{*} \square(\mathscr{S O}(2) \oplus \mathscr{S} \mathscr{U}(1,1)) .
\end{aligned}
$$

These two algebras are conjugated, which can be proved by considering the action of $\mathscr{S} \mathcal{O}(4,2)$ on the pseudo-euclidian space $E(4,2)$ and in fact turns to introduce the algebra

$$
\mathscr{I} \mathscr{S} \mathcal{O}(4,2)=\mathscr{T}(4+2) \square \mathscr{S O}(4,2) .
$$

$\mathscr{T}(4+2)$ is the 6-dimensional translational algebra and $\mathscr{S} \mathcal{O}(4,2)$ acts on its generators $V_{\mu}$ as

$$
\left[M_{\mu v}, V_{\varrho}\right]=i\left(g_{v} V_{\mu}-g_{\mu \varrho} V_{v}\right) .
$$

Then it can be shown that the 10 -dimensional algebra $\left(\tilde{\mathscr{S}}_{2}, \Lambda\right)$ is the stabilizer of the 2-plane $\left(V_{0^{\prime}}+V_{4}, V_{0}+V_{3}\right)$. Indeed one can verify that 
$\left(\tilde{\mathscr{S}}_{2}, \Lambda\right)$ is maximal in $\mathscr{S} \mathcal{O}(4,2)$ and by using $(9)$ that it stabilizes the above 2-plane. Identically $\left(\tilde{\mathscr{S}}_{2}^{*}, \Lambda\right)$ is the stabilizer of the 2-plane $\left(\boldsymbol{V}_{0^{\prime}}-\boldsymbol{V}_{4}\right.$, $\left.\boldsymbol{V}_{0}-\boldsymbol{V}_{3}\right)$.

One achieves the proof by checking that the two above 2-planes are conjugated [for example under the inner automorphism: $\boldsymbol{V} \rightarrow \exp \left(\boldsymbol{i} \pi \boldsymbol{M}_{34}\right)$ - $\left.\boldsymbol{V} \exp \left(-\boldsymbol{i} \pi \boldsymbol{M}_{34}\right)\right]$ which ensures that $\tilde{\mathscr{S}}_{2}$ and $\tilde{\mathscr{S}}_{2}^{*}$ are conjugated in the same way.

Hence the decomposition we have introduced permits to show the existence of an interesting chain of subalgebras between $\tilde{\mathscr{G}}_{2}$ and $\mathscr{S} \mathscr{O}(4,2)$ which does not contain the Poincare algebra and we can draw the following complete chains (we mean by complete a chain such that it does not exist any algebra between two adjacent algebras).

To be more precise we have represented by dotted lines the little chains which exist between the two physical chains, and it is worth noticing that there exists no other chain between $\tilde{\mathscr{S}}_{2}$ and $\mathscr{S} \mathcal{O}(4,2)$.

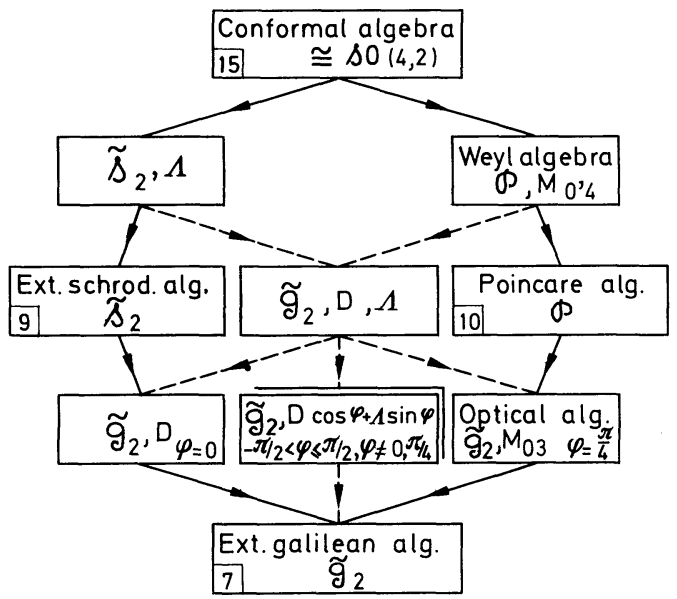

Physically the consequence of the inclusion of $\tilde{\mathscr{S}}_{2}$ in $\mathscr{S} \mathcal{O}(4,2)$ leads to associate to any relativistic massless particle a non-relativistic one with a variable mass in a two-dimensional space (the transverse plane). Indeed the d'Alembert equation $p^{2}|\rangle=0$, describing the states $\mid>$ of a massless relativistic particle in the momentum space, formally becomes $\left(\boldsymbol{H}-\frac{\boldsymbol{P}_{\perp}^{2}}{2 \boldsymbol{M}}\right)|\rangle=0$ with $\boldsymbol{P}_{\perp}=\left(\boldsymbol{P}_{1}, \boldsymbol{P}_{2}\right)$, for states such that $\boldsymbol{M}|\rangle \neq 0$, i.e., furnishes the Schrödinger equation describing the states of a free non-relativistic particle which is invariant under the Schrödinger algebra. 
Furthermore if we start with two non-relativistic massless particles $\boldsymbol{a}$ and $\boldsymbol{b}$. then $\boldsymbol{p}=\boldsymbol{p}(\boldsymbol{a})+\boldsymbol{p}(\boldsymbol{b})$ and we are led to the formal Schrödinger equation

$$
\left(\boldsymbol{H}-\frac{\boldsymbol{P}_{\perp}^{2}(\boldsymbol{a})}{2 \boldsymbol{M}(\boldsymbol{a})}-\frac{\boldsymbol{P}_{\perp}^{2}(\boldsymbol{b})}{2 \boldsymbol{M}(\boldsymbol{b})}+\boldsymbol{V}\right)|\rangle=0
$$

where $\boldsymbol{V}$ is a function of the relative transverse momentum $\boldsymbol{P}_{\perp}(\boldsymbol{a}, \boldsymbol{b})$

with

$$
V=\frac{M(a)+M(b)}{2 M(a) M(b)} P_{\perp}^{2}(a, b)
$$

$$
\boldsymbol{P}_{\perp}(\boldsymbol{a}, \boldsymbol{b})=\frac{\boldsymbol{M}(\boldsymbol{a}) \boldsymbol{P}_{\perp}(\boldsymbol{b})-\boldsymbol{M}(\boldsymbol{b}) \boldsymbol{P}_{\perp}(\boldsymbol{a})}{\boldsymbol{M}(\boldsymbol{a})+\boldsymbol{M}(\boldsymbol{b})} .
$$

This does not contradict the invariance under $\tilde{\mathscr{S}}_{2}$ since it has been shown in Ref. [5] that this invariance allows the existence of some classes of mutual interactions between the constituents of the free system.

In fact the existence of such a non-relativistic structure in the conformal algebra is subjacent in the wave packet realization of lightlike states given by Domokos [2].

In our formalism the definition of a lightlike state becomes

$$
\begin{aligned}
& \boldsymbol{P}_{\boldsymbol{j}}|\rangle=0, \quad \boldsymbol{j}=1,2, \\
& \boldsymbol{M}|\rangle=\boldsymbol{k}|\rangle
\end{aligned}
$$

where $\boldsymbol{k}$ is the color of the state. From the above commutation relations $(3,5,7,8)$ it is clear that the algebra of transformations which leave the color invariant, i.e. the algebra which commutes with $\boldsymbol{M}$ is exactly $\tilde{\mathscr{S}}_{2}$. The Domokos's lightlike states completely agree with our interpretation of the conformal algebra since the color is associated to the "mass" of the non-relativistic particle, but we must remark that the generator of "time" translations (the "Hamiltonian" $\boldsymbol{H}$ ) does not correspond to the compact subalgebra of $\mathscr{S} \mathscr{U}(1,1): \boldsymbol{H}+\boldsymbol{C}$ alone generates $\mathscr{S} \mathscr{O}(2)$, hence is able to have a discrete oscillatorlike spectrum [5].

The situation is rather different in what concerns the extreme collinear free massless quarks model introduced in Ref. [3]. The collinear states describing massless particles travelling in the $z$-direction are defined up to a conjugation by

$$
\begin{aligned}
& \boldsymbol{P}_{\boldsymbol{j}} \mid>=0, \quad \boldsymbol{j}=1,2, \\
& \boldsymbol{H}|\rangle=0 .
\end{aligned}
$$

But the used invariance notion of state space makes the model go out of our interpretation and leads to consider the algebra which in our formalism corresponds to $\boldsymbol{J}_{3}, \boldsymbol{D}, \boldsymbol{M}, \boldsymbol{M}^{*}, \Lambda$. As it has been above noticed 
$\boldsymbol{M}, \boldsymbol{M}^{*}, \Lambda$ form a non-compact algebra which corresponds to $\mathcal{O}(2,1)$ in Ref. [3], and it may be interesting to remark that their conformal quantum number is the eigenvalue of the compact generator $\boldsymbol{M}-\boldsymbol{M}^{*}$ which turns to account the "masses" of the two Heisenberg algebras present in the decomposition of $\mathscr{S O}(4,2)$ we have given.

We hope that this brief analysis brings some insight about the point raised in the footnote (9) of the Ref. [3] in the following sense: the Domokos's approach uses the underlying non-relativistic analogy contained in the conformal algebra, while the invariance notion retained by Del Giudice et al. discards this analogy.

In conclusion we lay stress on the scheme we have drawn which clearly makes appear the double, - relativistic and non-relativistic -, content of the conformal algebra and the conformal nature of the Schrödinger algebra.

Finally let us add that the same kind of scheme can be drawn between the algebra $\mathscr{S} \mathcal{O}(5,2)$ and the 3-dimensional Galilean algebra embedded on one side in the Schrödinger algebra $\tilde{\mathscr{S}}_{3}$, on the other side in the Poincaré algebra $\mathscr{P}(4,1)$ corresponding to transformations on the $\mathscr{M}(4,1)$ Minkowski space.

\title{
References
}

1. Bouchiat, C., Fayet,P., Meyer,Ph.: Nucl. Phys. B 34, 157 (1971)

Meyer, Ph.: Cargese's Lectures (1972), and all the references about the use of $\tilde{\mathscr{G}}_{2} \subset \mathscr{P}_{2}$ given therein

2. Domokos, G.: Commun. math. Phys. 26, 15 (1972)

3. Del Giudice,E., Di Vecchia,P., Fubini, S., Musto, R.: Nuovo Cimento 12 A, 813 (1972)

4. Niederer, U.: Helv. Phys. Acta 45, 802 (1972)

Roman,P., Aghassi, J.J., Santilli, R.M., Huddelston,P.: Nuovo Cimento 12 A, 185 (1972)

Burdet, G., Perrin, M., Sorba, P.: Preprint Marseille 72/p. 499 (1972)

5. Burdet, G., Perrin, M.: Lettere Nuovo Cimento 4, 651 (1972)

\author{
G. Burdet \\ CNRS-Centre de Physique Théorique \\ 31 Chemin J. Aiguier \\ F-13274 Marseille Cedex 2, France
}

